\title{
Point scatterer enhancement in ultrasound by wavelet coefficient shrinkage
}

Stine M. Hverven, Ole Marius Hoel Rindal, Alan J. Hunter and Andreas Austeng

Citation: 2017 IEEE International Ultrasonics Symposium (IUS), Washington, DC, 2017, pp. 1-4; doi: 10.1109/ULTSYM.2017.8092971

Published by: Ultrasonics Symposium (IUS), 2017 IEEE International 


\title{
Point scatterer enhancement in ultrasound by wavelet coefficient shrinkage
}

\author{
Stine M. Hverven*, Ole Marius Hoel Rindal*, Alan J. Hunter ${ }^{\dagger *}$ and Andreas Austeng* \\ *Department of Informatics \\ University of Oslo \\ Oslo, Norway \\ Email: stinemhv@ifi.uio.no \\ †University of Bath \\ Bath, United Kingdom
}

\begin{abstract}
Background speckle can often obscure objects of interest in an ultrasound image. The probability of detection and classification of point scatterers is highly affected by background speckle. The proposed algorithm uses a coherence-based wavelet coefficient shrinkage method. Point scatterers in the ultrasound image are enhanced by separating coherent point targets from incoherent background speckle. Results using Field II ultrasound simulations show how the algorithm retains the point scatterers and increases their conspicuity. The algorithm has potential to detect microcalcifications in breast tissue.
\end{abstract}

Keywords-Point scatterer, Wavelet shrinkage, Microcalcifications, Coherence, Ultrasound.

\section{INTRODUCTION}

The size, distribution and morphology of microcalcifications in breasts can for some cases be considered an early indicator of breast cancer. Microcalcifications are small, hard calcium deposits in soft breast tissue and behave as point scatterers in an ultrasound image. However, detection of microcalcifications in ultrasound images is challenging as they are often obscured by background speckle.

The proposed algorithm uses a coherence-based wavelet shrinkage method to suppress speckle background in order to enhance point scatterers. From the original image, the algorithm creates multiple images or looks with statistically independent noise realizations and retained wavenumber resolution. The algorithm then utilizes a coherence metric to determine the similarity of the wavelet coefficients between these looks. The wavelet coefficients with low coherence are attenuated to produce an image with reduced speckle. The method has previously been applied on sonar images to separate coherent targets from incoherent background reverberation noise [1].

\section{METHODS}

\section{A. Phantom Data Sets}

A phantom with three point scatterers in speckle was designed to test the algorithm's ability to enhance point scatterers. The simulation for the point scatterers and the speckle were run separately in order to vary the speckle intensity level more easily. Each data set was normalized by its maximum intensity, but the speckle data was further intensity scaled relative to the point scatterers using the relative values of 50, 70 , and $95 \%$. The scaled speckle data was then added to the other data to create images with varying speckle intensity. The synthetic aperture dataset was simulated using Field II [2][3] and a linear array with 128 elements, $5 \mathrm{MHz}$ center frequency and $\lambda$ pitch (properties of Philips L11-4v). Beamforming was performed using The Ultrasound Toolbox (USTB) [4].

The method was also verified on an ultrasound image of a real tissue-mimicking phantom (CIRS 054GS) with a hyperechoic area and several point scatterers. A coherently compounded plane wave ultrasound image was acquired with the Verasonics Vantage system using a linear probe (Philips L7-4, 128 elements, 75 angles, 5.2 MHz, f\# = 1.75). As with the simulated data, an image of only speckle was also recorded and used to create an image with extra $70 \%$ speckle intensity.

\section{B. Algorithm Description}

Fig. 1 shows a flowchart of the algorithm. The edges of the original image are first tapered down to reduce Gibbs phenomenon in the discrete Fourier transform of the images. Two complimentary looks are then generated as described below. These looks are subsequently decomposed into wavelet coefficients. The coherence between the wavelet transforms is estimated, and after $p$ realizations an averaged estimate of the coherence is thresholded. This is multiplied with the wavelet transform of the original image to generate the final image.

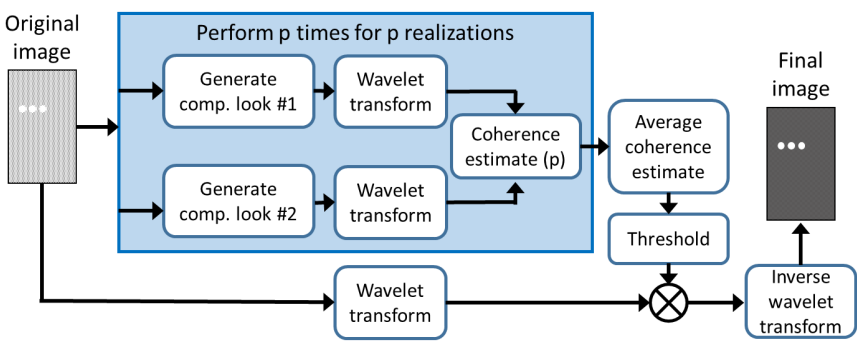

Fig. 1. Flowchart of the wavelet coefficient shrinkage algorithm.

Complimentary looks of the original image are first generated by partitioning the frequency space, i.e. the 2D FFT of the image into two sets. This is accomplished by applying a 2D grid to the space where blocks are randomly assigned to either of the two sets, see Fig. 2. The size of the blocks in the grid is chosen so that information of the point scatterers is preserved over several blocks, while the speckle background is correlated on a much smaller scale than the block size [1]. The point scatterers will then be persistent in both of the resulting two complimentary looks, whereas the speckle will ideally not have high coherence between the looks. 


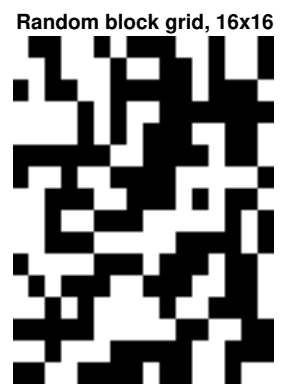

(a) Block grid

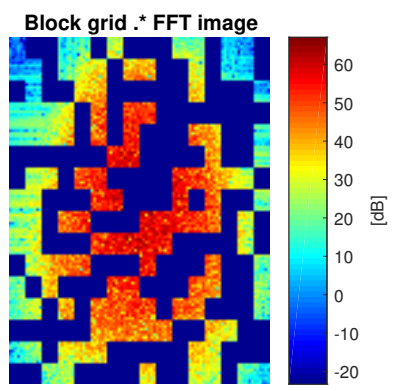

(b) Partitioned FFT
Fig. 2. Generation of complimentary looks by partitioning frequency space using a random block grid (a). The partitioned frequency space shown here (b) creates one look, and the other is created using the inverse block grid.

Wavelets can be used to de-noise images while preserving resolution. The target information can be described using a few wavelet coefficients, whereas noise is more evenly distributed [1]. Thresholding these wavelet coefficients will give a final inverse wavelet transformed image which has retained target information and suppressed noise.

The multilevel 2D wavelet decomposition was performed with a symmetric biorthogonal wavelet base [5]. The discrete wavelet transform decomposes an image into high and low frequencies. From the high-pass filtered image, three detail images are created, each describing directional local changes in the image. The low-pass filtered image is further downscaled and yields what is known as an approximation image. This image is further high-pass filtered to produce the three smaller detail images of the next level, and so on.

The coherence between two looks is estimated as:

$$
\mathrm{C}(x, y, l, p)=\left|\frac{\sum_{m, n=-\frac{N-1}{2}}^{\frac{N-1}{2}} \mathrm{~W}_{1} \mathrm{~W}_{2}^{*}}{\sqrt{\sum_{m, n=-\frac{N-1}{2}}^{\frac{N-1}{2}}\left|\mathrm{~W}_{1}\right|^{2}} \sqrt{\sum_{m, n=-\frac{N-1}{2}}^{\frac{N-1}{2}}\left|\mathrm{~W}_{2}\right|^{2}}}\right|
$$$$
\text { given } \mathrm{W}_{i}=\mathrm{W}_{i}(x+m \Delta x, y+n \Delta y, l, p)
$$

where $(x, y)$ is the pixel location in the wavelet transformed image, $l$ is the decomposition level, $p$ is the realization number, and $\Delta x$ and $\Delta y$ are the pixel dimensions. $\mathrm{W}_{i}$ is the wavelet transform of complementary look $i$ and $\mathrm{W}_{i}^{*}$ denotes its conjugate. $N \mathrm{x} N$ is the sliding window size over which the coherence for pixel location $(x, y)$ is calculated. A hanning window with $N=5$ was used. A large window size will reduce variance, but also reduce spatial resolution. The average coherence estimate for each level $l$ is obtained by averaging the coherence estimate between two looks over all $P$ realizations.

$$
\mathrm{C}_{\mathrm{av}}(x, y, l)=\frac{1}{P} \sum_{p=1}^{P}|\mathrm{C}(x, y, l, p)|
$$

The average coherence estimate is thresholded by a simple weighting scheme where coefficients with values less than $t_{\text {min }}$ are completely suppressed, values greater than $t_{\max }$ are retained, and between these two there is a linear transition.

$$
\mathrm{C}_{\mathrm{th}}(x, y, l)= \begin{cases}1, & \text { if } C_{\mathrm{av}}(x, y, l)>t_{\mathrm{max}} \\ 0, & \text { if } C_{\mathrm{av}}(x, y, l)<t_{\mathrm{min}} \\ \frac{\mathrm{C}_{\mathrm{av}}(x, y, l)-t_{\min }}{t_{\max }-t_{\min }}, & \text { otherwise }\end{cases}
$$

These thresholded coefficients are multiplied with the wavelet coefficients of the original image, and the inverse wavelet transform of the adjusted coefficients yield the final image.

\section{Evaluation Criteria}

As a visibility metric for the point scatterers, the metrics conspicuity $(\mathrm{Cp})$ and $\mathrm{Peak}_{\text {point }}$-to-Peak $\mathrm{speckle}_{\text {satio }}(\mathrm{PP})$ are presented. $\mathrm{Cp}$ is a measure of how clearly discernible a point is from the background at same depth and it is defined as [6]:

$$
\text { Conspicuity }=\frac{\max _{\text {point }}-\mu_{\text {speckle }}}{\sigma_{\text {speckle }}} .
$$

PP measures the intensity difference between the point scatterers and peaks in the speckle background:

$$
\mathrm{PP}=20 \log _{10}\left(\frac{\max _{\text {point }}}{\text { max }_{\text {speckle }}}\right)
$$

where $\max _{\text {point }}$ and $\max _{\text {speckle }}$ are the maximum intensity values. We call $\mathrm{PP}_{1}$ the ratio calculated within the areas depicted by the solid white lines in Fig. 3, i.e. areas at same depth. $\mathrm{PP}_{2}$ is calculated when the maximum speckle intensity is within the area depicted by the dotted white lines.
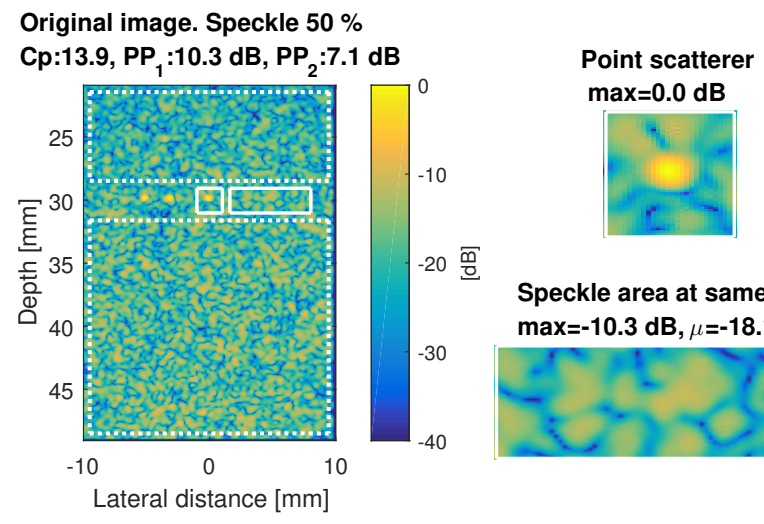

Fig. 3. Conspicuity $(\mathrm{Cp})$ and $\mathrm{Peak}_{\text {point }}$-to-Peak speckle ratio (PP) metrics calculated using (4) and (5).

\section{RESULTS}

Fig. 4 shows the results when the method is applied to a plane wave image of a real tissue-mimicking phantom. Conspicuity of the point scatterers in the wavelet shrunk image is around 14-17 times higher than in the original. A more detailed analysis is presented for the simulated phantom.

The threshold levels for the simulated phantom were chosen based on the average coherence estimates for each level, shown in Fig. 5. The three point scatterers are easily discernible in the first level decomposition images with coherence values around 0.6. The points are somewhat discernible in the second level with coherence values above 0.35 . Further decomposition does not include much of the target information. 

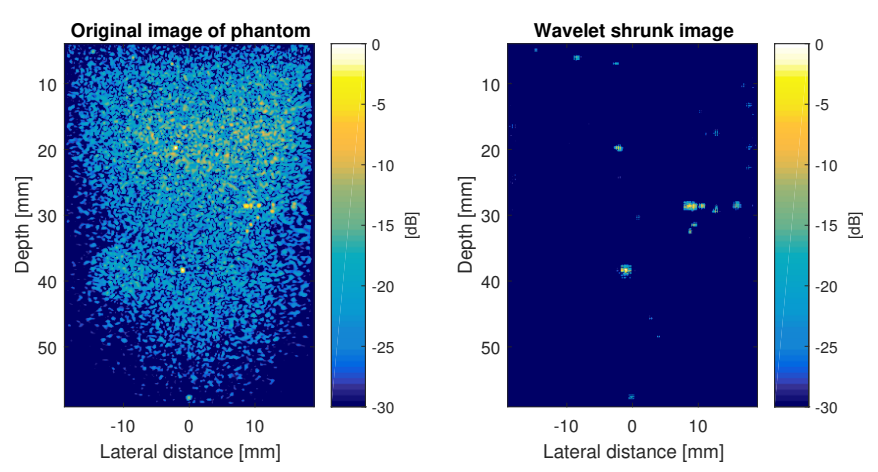

Fig. 4. Results on an image of a real tissue-mimicking phantom. $\mathrm{Cp}$ is $14-17$ times higher in the wavelet shrunk image.

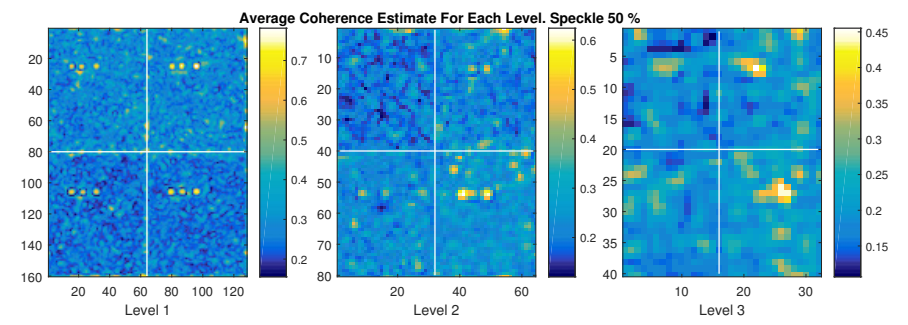

Fig. 5. Average coherence estimates for the simulated phantom. The four coefficient images are shown together for each wavelet decomposition level. The upper left corner shows the approximation image. The other three corners show the three detail images; horizontal (top right), vertical (bottom left) and diagonal (bottom right).

The approximate coherence values found in Fig. 5 were used as initial threshold limits and were further adjusted. Mild threshold limits are more likely to retain all point scatterers, but will also include more of the speckle background and increase the possibility of false positives in the final image. Three different threshold limits were used to create the thresholded average coherence estimates shown in Fig. 6.

TABLE I. CP AND PP MEASUREMENTS CORRESPONDING TO FIG. 7 , CALCULATED USING (4) AND (5).

\begin{tabular}{|c|c|c|c|c|c|c|}
\hline Speckle & \multicolumn{2}{|c|}{ Cp } & \multicolumn{2}{c|}{ PP $_{1}$} & \multicolumn{2}{c|}{ PP $_{2}$} \\
\cline { 2 - 7 }$[\%]$ & Original & Final & Original & Final & Original & Final \\
\hline $\mathbf{5 0}$ & 13.9 & 247.9 & $10.3 \mathrm{~dB}$ & $33.3 \mathrm{~dB}$ & $7.1 \mathrm{~dB}$ & $18.3 \mathrm{~dB}$ \\
$\mathbf{7 0}$ & 9.3 & 301.2 & $7.3 \mathrm{~dB}$ & $35.7 \mathrm{~dB}$ & $4.1 \mathrm{~dB}$ & $12.3 \mathrm{~dB}$ \\
$\mathbf{9 5}$ & 6.2 & 359.4 & $4.6 \mathrm{~dB}$ & $36.6 \mathrm{~dB}$ & $1.4 \mathrm{~dB}$ & $6.5 \mathrm{~dB}$ \\
\hline
\end{tabular}

Fig. 7 shows the results from the simulated phantom when the speckle background intensity is varied (50,70 and $95 \%$ ). All three point scatterers are retained when their intensity is above maximum speckle intensity, and the conspicuity values are around 18-58 times larger than in the original image. Table I summarizes these results. Fig. 8 shows the results when using two different threshold limits for the $50 \%$ speckle image.

\section{Discussion}

Results on both the tissue-mimicking phantom in Fig. 4 and the simulated data in Fig. 7 show how the method can suppress speckle background. From Fig. 6 we expect best results when threshold values for the first and second level are relatively strict, since this excludes coefficients describing only speckle background. Decomposing with mild threshold limits to more than three levels resulted in the inclusion of more background
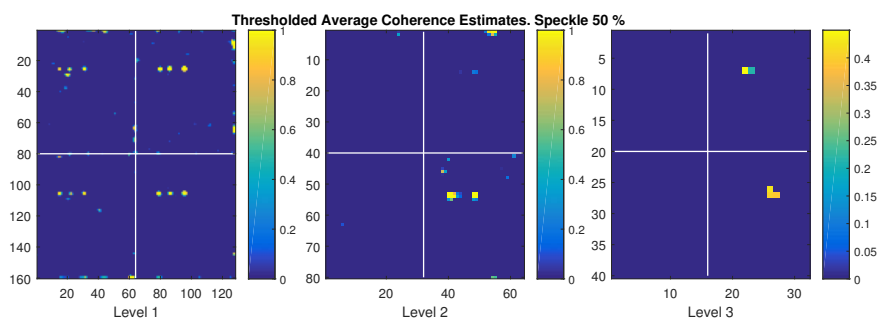

(a) Threshold limits: $(0.5-0.65,0.45-0.55,0.4-0.5)$. Speckle $50 \%$

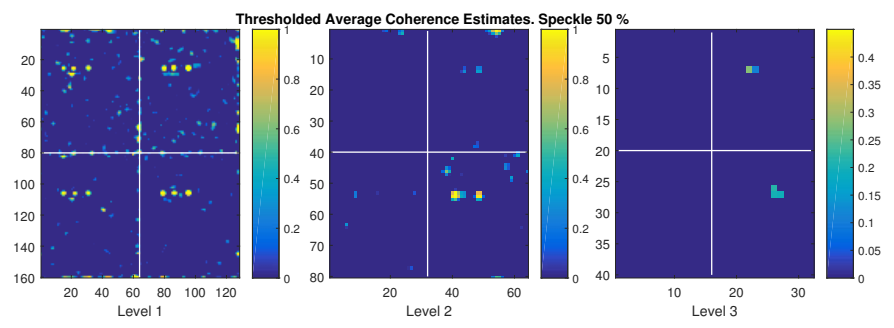

(b) Threshold limits: (0.4-0.6, 0.4-0.6, 0.4-0.6). Speckle $50 \%$

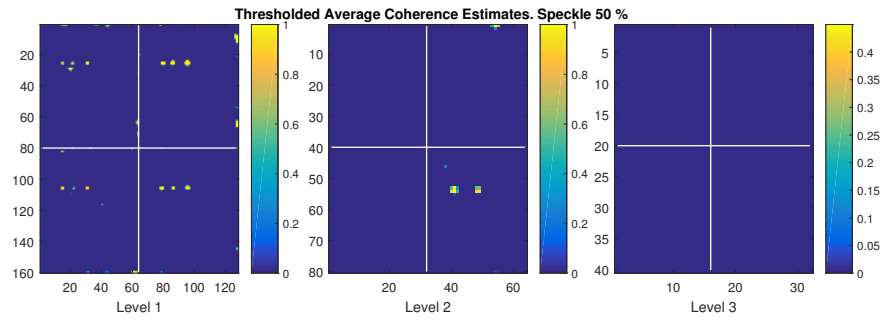

(c) Threshold limits: $(0.55-0.65,0.5-0.6,0.5-0.6)$. Speckle $50 \%$

Fig. 6. Thresholded average coherence estimates using different threshold limits, applied on the images in Fig. 5. The top image (a) corresponds to results shown in Fig. 7a, and (b) and (c) correspond to Fig. 8.

speckle in the final image. From results shown in Fig. 8 we see that the best result for the $50 \%$ speckle intensity is obtained when the threshold limits are so strict that the last level is completely attenuated, corresponding to an actual two-level decomposition.

Results presented in Fig. 7 and summarized in Table I illustrate how the algorithm increases conspicuity by around 18-58 times more than in the original image. The method retains all of the point scatterers, even when intensity peaks in the speckle background are almost at the same level. When the speckle intensity increases, the chance of false positives in the final image also increases. The high speckle values in the shallow and deep depth range seen in the $95 \%$ speckle results are assumed edge artifacts caused by Gibbs effect.

The results show it is essential to choose threshold limits for each level based on the coherence estimate values, which in turn are related to the size of the target objects and the intensity of the speckle background in the original image. When the speckle intensity is almost at the same level as the point scatterers, a strict thresholding can exclude too much information. With high relative speckle intensity, the method can be beneficial as a supplement to the conventional ultrasound image.

It is expected that a large number of realizations reduces the variance in the final image. However, results showed no difference between using $\mathrm{P}=100$ or $\mathrm{P}=10000$ realizations. $\mathrm{P}=12$ 

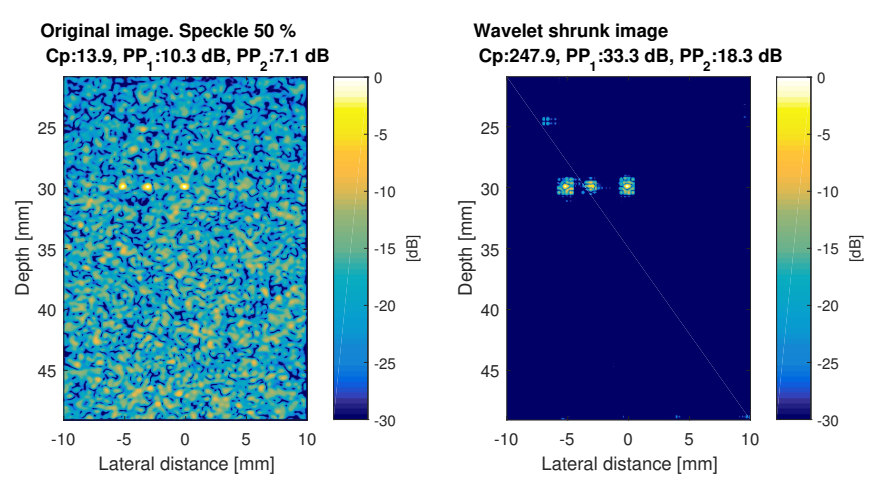

(a) Speckle $50 \%$. Threshold limits: $(0.5-0.65,0.45-0.55,0.4-0.5)$
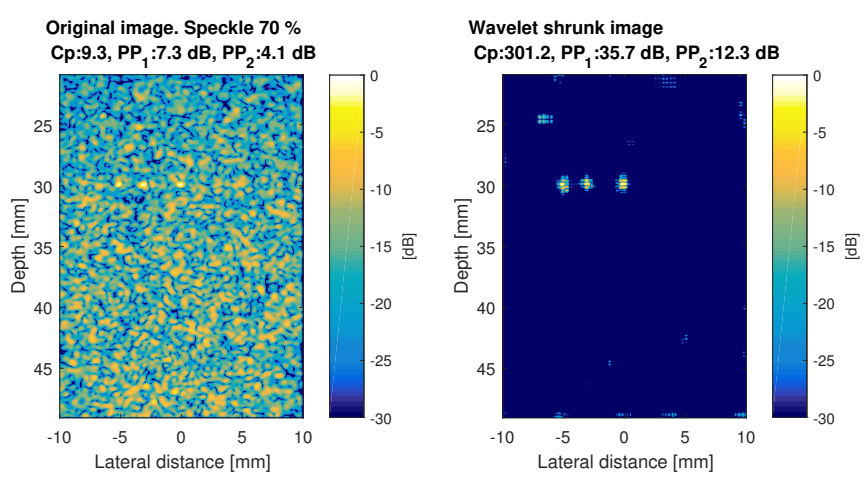

(b) Speckle $70 \%$. Threshold limits: $(0.5-0.65,0.45-0.55,0.4-0.5)$
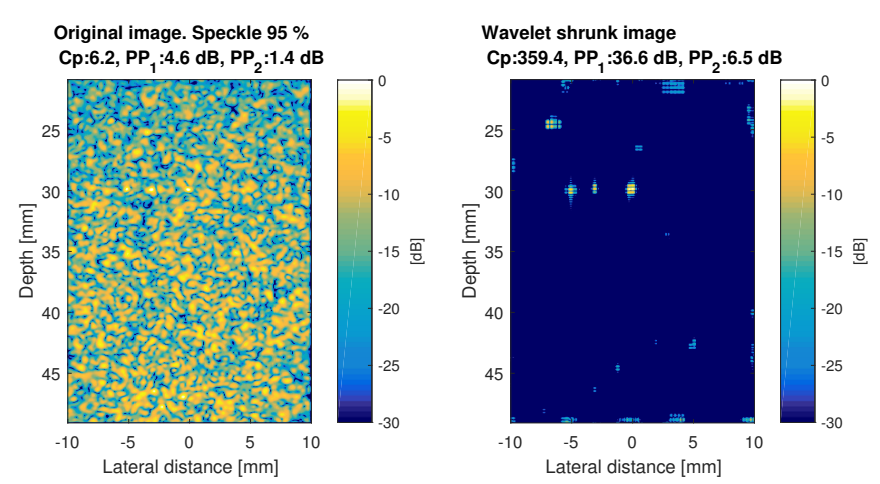

(c) Speckle $95 \%$. Threshold limits: (0.5-0.65, 0.45-0.55, 0.4-0.5)

Fig. 7. Results with varying speckle background intensity (50, 70 \& $95 \%$ ). $\mathrm{Cp}$ and PP measurements are summarized in Table I.

was similar to $\mathrm{P}=100$ when using the same parameters. When $\mathrm{P}$ was reduced to six realizations a little more of the speckle background was visible in the final image. This indicates that few realizations are required to produce a final image with well suppressed speckle background. The results when varying the threshold limits show how it is also not necessary to decompose into many levels and that even two levels might suffice. The fact that the algorithm works well with a low number of realizations and few levels is very positive for its computation time. The initial results indicate that the algorithm can enhance point scatterers that are almost buried in speckle, a situation quite similar to microcalcifications in breasts.

\section{CONCLUSIONS}

We have shown that the wavelet coefficient shrinkage algorithm manages to suppress speckle background and enhance
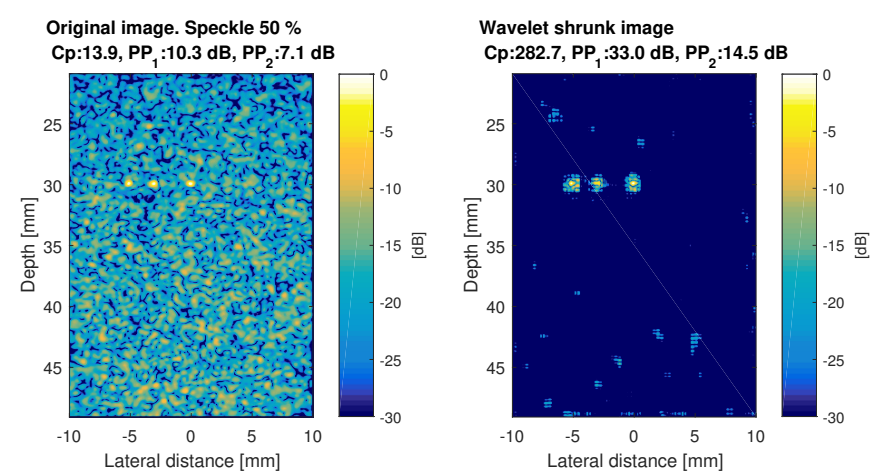

(a) Results using threshold limits: (0.4-0.6, 0.4-0.6, 0.4-0.6).
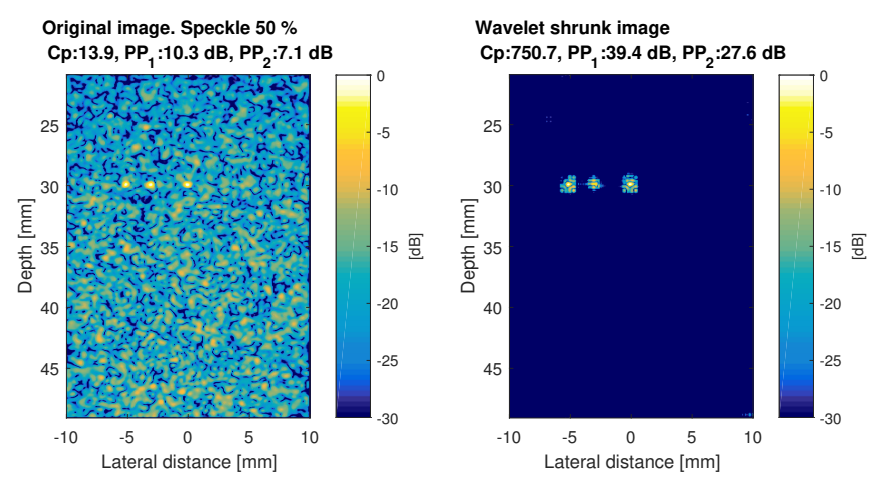

(b) Results using threshold limits: (0.55-0.65, 0.5-0.6, 0.5-0.6).

Fig. 8. Results when applying the threshold limits shown in Fig. $6 \mathrm{~b}$ and $6 \mathrm{c}$.

point scatterers in an ultrasound image. The conspicuity of the point scatterers are greatly increased. The method retains the point scatterers even when intensity peaks in the speckle background are almost at the same intensity level. The method was tested for a variety of wavelet decomposition levels, thresholding schemes and number of realizations. Results show that few levels and number of realizations are necessary. The algorithm has potential to detect microcalcifications in breast tissue.

\section{REFERENCES}

[1] A. J. Hunter and R. van Vossen, "Sonar target enhancement by shrinkage of incoherent wavelet coefficients," The Journal of the Acoustical Society of America, vol. 135, no. 1, pp. 262-268, 2014.

[2] J. A. Jensen and N. B. Svendsen, "Calculation of Pressure Fields from Arbitrarily Shaped, Apodized, and Excited Ultrasound Transducers," IEEE Transactions on Ultrasonics, Ferroelectrics and Frequency Control, vol. 39, no. 2, pp. 262-267, 1992.

[3] J. A. Jensen, "Field: A Program for Simulating Ultrasound Systems," Medical \& Biological Engineering \& Computing, vol. 34, pp. 351-353, 1996.

[4] A. Rodriguez-Molares, O. M. H. Rindal, O. Bernard, A. Nair, M. A. Lediju Bell, H. Liebgott, A. Austeng, and L. Løvstakken, "The UltraSound ToolBox (USTB)," IEEE International Ultrasonics Symposium, IUS, 2017.

[5] I. Daubechies, "Ten Lectures on Wavelets," Society for Industrial and Applied Mathematics, pp. 251-287, 1992.

[6] M. A. Lediju, Trahey, G. E., Byram, B. C., and J. J. Dahl, "Short-lag spatial coherence of backscattered echoes: Imaging characteristics." IEEE Transactions on Ultrasonics, Ferroelectrics, and Frequency Control, vol. 58, no. 7, pp. 1377-1388, 2011. 